\title{
Indiscriminate ssDNA cleavage activity of CRISPR-Cas12a induces no detectable off-target effects in mouse embryos
}

\section{Dear Editor,}

Newly discovered characteristics like "collateral effect" or trans-cleavage in CRISPR-Cas 13 and CRISPR-Cas12 systems have enabled their usage in nucleic acid detection (Gootenberg et al. 2017, 2018; Chen et al. 2018). The collateral RNA cleavage of Cas13a has been reported to be harmful for cell development (Wang et al. 2019; Buchman et al. 2020). As a representative gene editor of CRISPRCas12 system, CRISPR-Cas12a (Cpf1) holds great potential for therapeutic applications in the future (Zetsche et al. 2015; Koo et al. 2018; Campa et al. 2019). However, when used for genome editing in mammalian cells, target-activated Cas $12 \mathrm{a}$ has the risk to cleave transiently exposed ssDNA during replication, transcription and homology-directed repair processes (Chen et al. 2018) (Fig. 1A), raising the concern of its therapeutic applications. Therefore, the potential off-target effects caused by the indiscriminate ssDNA cleavage activity of Cas12a need to be carefully investigated.

Recently, we developed a new approach called GOTI (Genome-wide Off-target analysis by Two-cell Injection) to detect off-target effects without the interference of singlenucleotide polymorphisms (SNPs) in individuals (Zuo et al. 2019). In this study, we designed an optimized method called genome-wide off-target analysis by twin blastomeres (GOAT) for off-target edits detection. Briefly, mouse embryos were separated into two embryos at two-cell stage, and then gene editing tools such as BE3, ABEmax and Cas12a were injected into one of the twin embryos (Figs. 1B and $\mathrm{S} 1 \mathrm{~A}$ ). To increase the pregnancy efficiency, twin embryos were co-transferred with two ICR embryos to the pseudopregnant mouse. When the twin embryos developed to embryonic day 12.5 (E12.5), twin embryos and ICR embryos were distinguished by their eye colors and a SNP site on Tyr gene (Fig. S1B and S1C). The edited embryo was distinguished from the unedited twin embryo by high editing efficiency to induce indels and nucleotide substitutions on the target sites. Whole-genome sequencing (WGS) was performed on the genomic DNA of twin embryos, separately. Then single-nucleotide variants (SNVs) and indels were called in the injected sample, with its twin un- injected one as the reference (Figs. 1B and S1A). GOAT could distinguish the injected and un-injected embryos directly, while GOTI relies on massive FACS to separate edited cells from unedited cells. In addition, GOAT could also avoid the leak of two-cell injection, false-negative FACS sorting and inferior developmental competition ability of the injected blastomere.

To test the effectiveness of GOAT system, we included three groups in our study: GFP, BE3, and ABEmax groups (Fig. S1A). The developmental rate of twin embryos to blastocysts was more than $90 \%$, and twin embryos developed to E12.5 was $23.0 \% \pm 3.1 \%(n=5$; Table S1). WGS was conducted separately for the twin embryos at an average depth of 30 to confirm on-target editing efficiency and analyze the potential genome-wide off-target effects (Table S2). The activities of BE3 and ABEmax were confirmed by the high on-target efficiencies to introduce nucleotide substitutions (Figs. S1D, S2 and S3).

For the off-target effects, we found $14 \mathrm{SNVs}$ and 0 indel per embryo on average in the GFP-injected group (Figs. 1C, 1D, S4 and Tables S3, S4, S5). For the BE3-injected embryos, we found 210 SNVs per embryo on average, 15 times more than those of the GFP group $(P=0.0025$; Figs. 1C, S4 and Tables S3, S6). By contrast, indels showed no differences between BE3 and GFP groups (Fig. 1D). We observed that about $86 \%$ of SNVs were mutated from $C$ to $T$, or $G$ to $A$ (Figs. 1E and S5), consistent with the results of GOTI method (Zuo et al. 2019). We also analyzed the offtarget effects of ABEmax using GOAT. An average of 18 SNVs and 0 indel were detected in each embryo, similar to the number found in the GFP-injected group $(P=0.57$; Figs. 1C, 1D, S4 and Tables S3, S4). Together, these results suggest that GOAT is a comparable approach to detect genome-wide off-target effects in mouse embryo comparing with GOTI.

We further used GOAT to analyze the genome-wide offtarget effects of two commonly used Cas12a (LbCas12a and AsCas12a). Similarly, LbCas12a or AsCas12a mRNA and their crRNAs targeting Dmd or Tp53 gene were injected into one of the twin embryos (Fig. 1B). The activities of LbCas12a and AsCas12a were confirmed by the high efficiency 
A

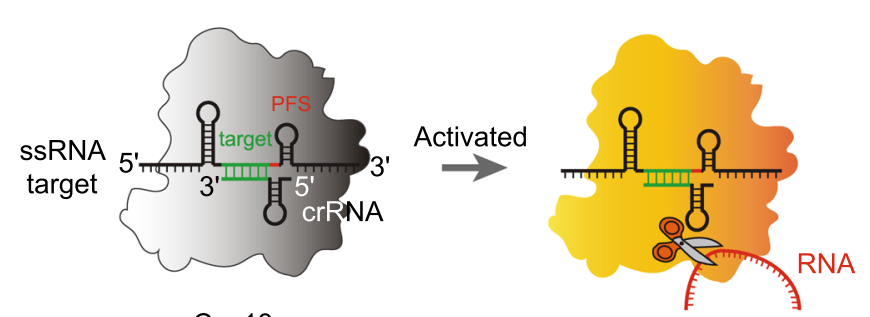

Cas13a

Promiscuous RNAse activity

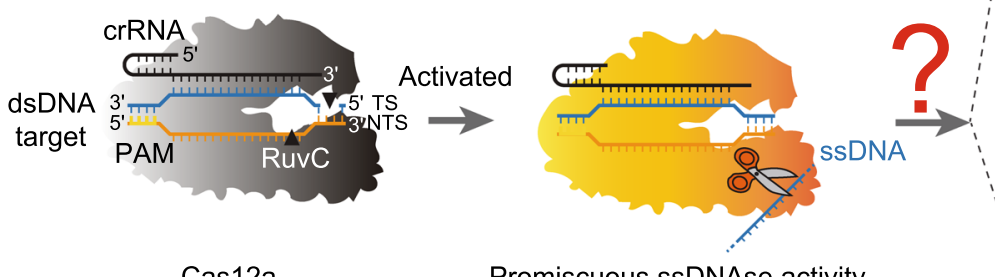

Cas12a

Promiscuous ssDNAse activity

B

Base editors/Cas12a + gRNA
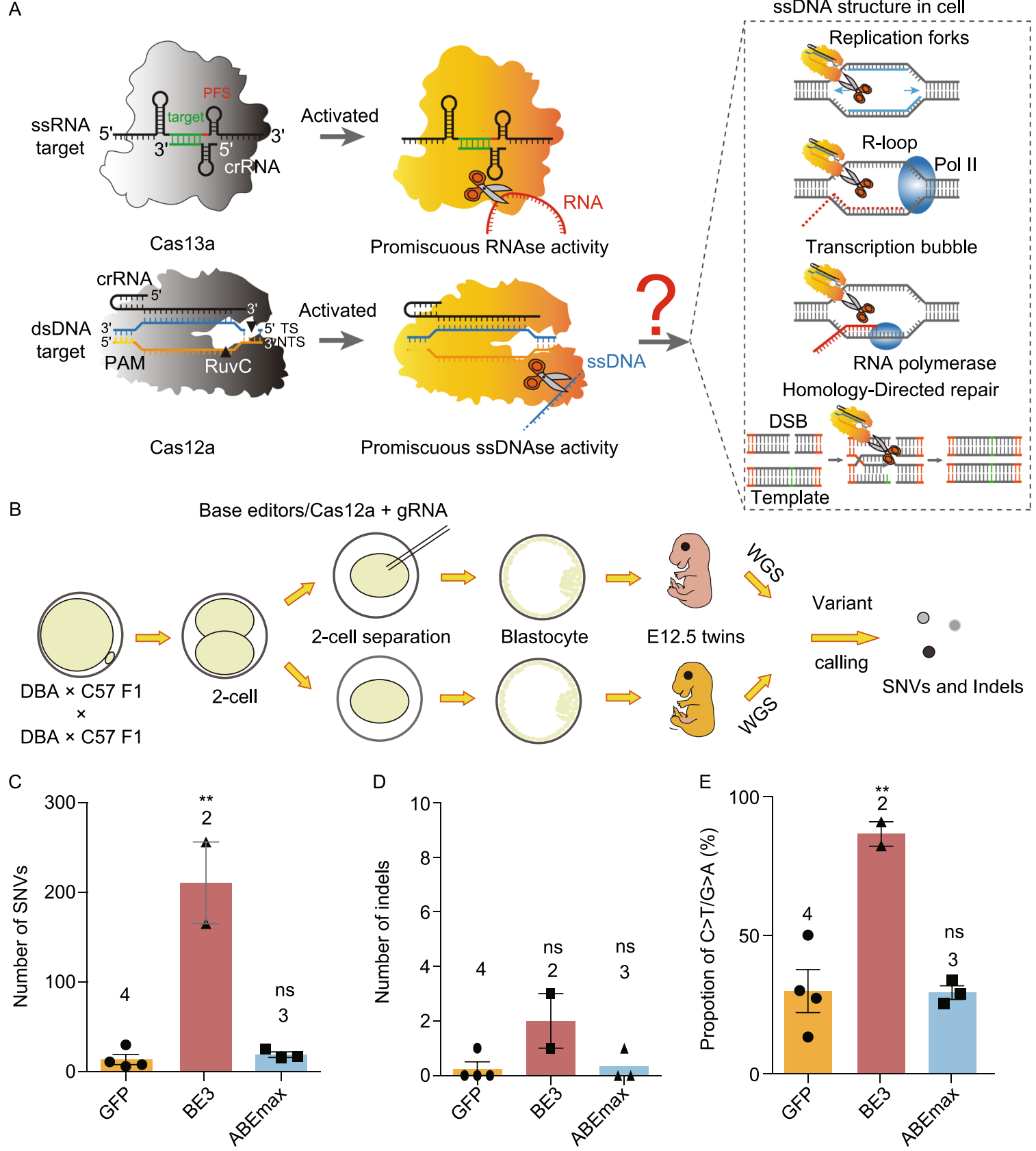

Figure 1. GOAT detects off-target effects induced by BE3, ABEmax and indiscriminate ssDNA cleavage activity of CRISPRCas12a. (A) "Collateral effect" or trans-cleavage in CRISPR-Cas13 and CRISPR-Cas12 systems. DNA replication, transcription, homology-directed repair and R loop structure would lead to the unwinding of double-stranded DNA (dsDNA) to ssDNA. Whether the indiscriminate ssDNA cleavage activity of Cas12a would induce genome-wide off-target effects in mammalian cells needs to be explored. (B) Experimental design of GOAT mediated genome-wide off-target detection. (C) Number of SNVs identified in GFP, BE3, and $A B E m a x$ injected groups. (D) Number of indels identified in GFP, BE3, and ABEmax injected groups by WGS. (E) The proportion of G.C to A.T mutations in GPF-, ABEmax-, and BE3-injected groups. Numbers above the columns represent the number of samples. $n=4$ for GFP, $n=3$ for BE3 and $n=3$ for ABEmax groups. All values are presented as mean \pm SEM. ${ }^{\star} P<0.05$, ${ }^{\star \star} P<0.01$, ${ }^{\star * \star} P<0.001$, ns, $P \geq 0.05$, unpaired $t$-test.

(100.0\% $\pm 0.0 \%$ for LbCas $12 a-D m d, 100.0 \% \pm 0.0 \%$ for AsCas12a-Dmd; $83.3 \% \pm 16.7 \%$ for LbCas $12 a-P 53,100.0 \%$ $\pm 0.0 \%$ for AsCas12a-P53; $n=3$ twins for each group) to induce indels on Dmd gene. (Figs. 2A and S2). We found an average of 19 SNVs and 1 indel in LbCas12a group. Similarly, 18 SNVs and 1 indel were detected in AsCas12a group 
A
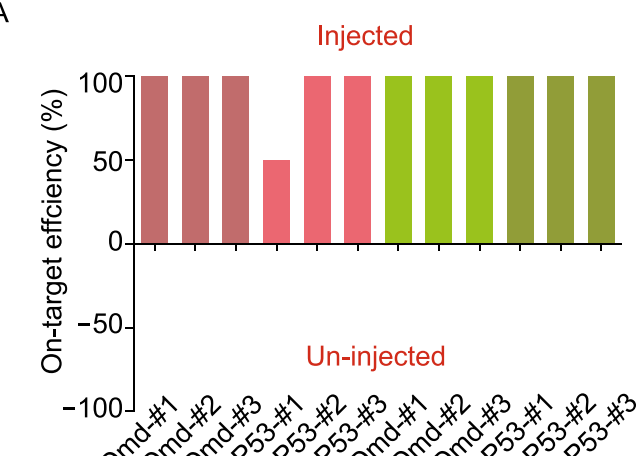

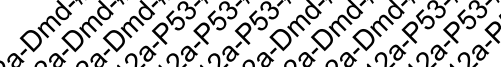

$2^{2}+2^{2}+2^{2}+2^{2}+2^{2}+2^{2}+2^{2}+2^{2}, 2^{2}+2^{2}+2^{2}, 2$

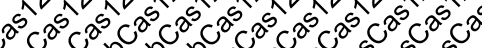

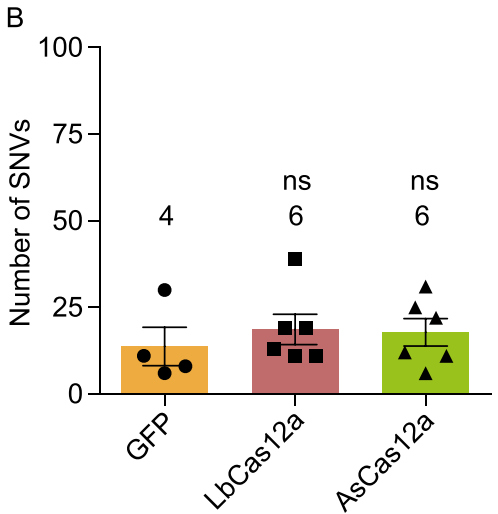

C

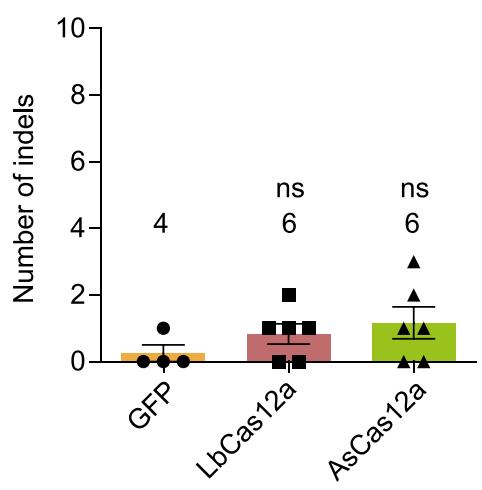

D

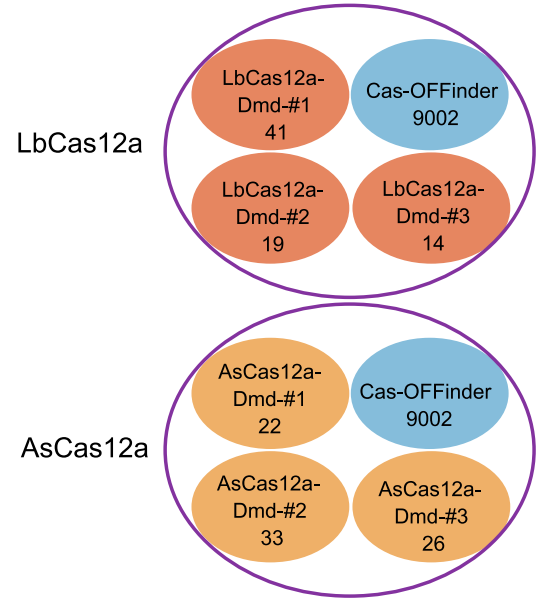

$\mathrm{F}$

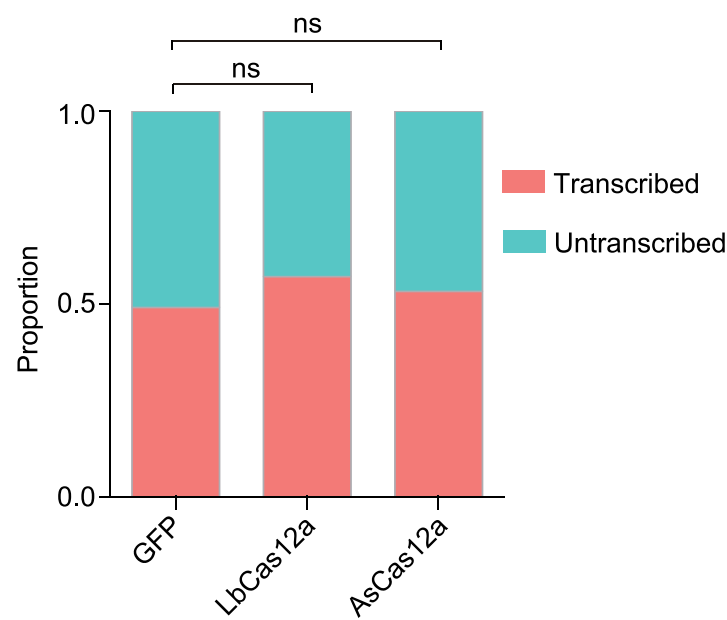

$E$

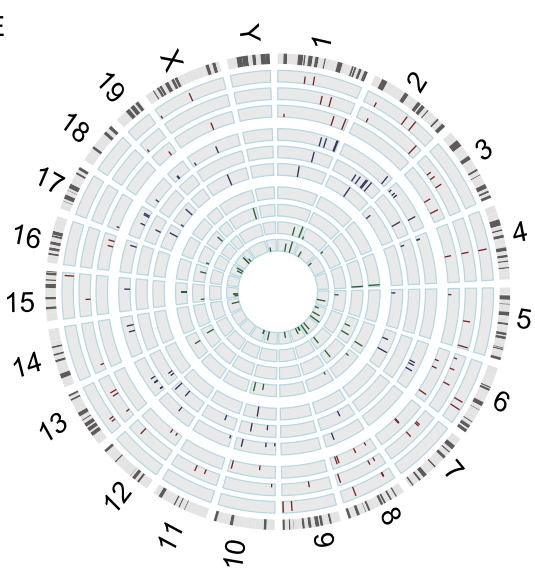

GFP LbCas12a-Dmd AsCas12a-Dmd

G

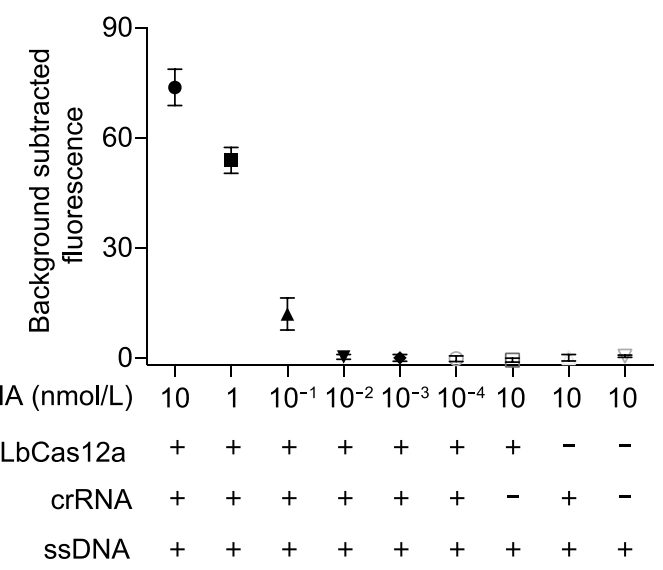


Figure 2. On-target editing and off-target effects of LbCas12a and AsCas12a using GOAT. (A) On-target editing efficiency identified by WGS for LbCas12a and AsCas12a groups. (B) Number of SNVs identified in LbCas12a and AsCas12a groups by WGS, where on-target editing was removed from the analysis. (C) Number of indels identified in LbCas12a and AsCas12a groups by WGS. (D) Overlap among SNVs and indels detected by GOAT with predicted off-targets by Cas-OFFinder. (E) Distribution of SNVs in the mouse genome in GFP, LbCas12a-treated and AsCas12a-treated samples. Embryos from inner circle to outer circle were GFP\#1, GFP-\#2, GFP-\#3, LbCas12a-Dmd-\#1, LbCas12a-Dmd-\#2, LbCas12a-Dmd-\#3, AsCas12a-Dmd-\#1, AsCas12a-Dmd-\#2 and AsCas12a-Dmd-\#3. (F) The distribution of off-target SNVs in the transcribed and un-transcribed regions. $P$ values were calculated by Chi-square test on the number of SNVs in transcribed and un-transcribed regions of each group. (G) Correlation between the target DNA dosage and the presence of cleaved ssDNA. $n=4$ twins for GFP, $n=6$ twins for LbCas12a and $n=6$ twins for AsCas12a groups; numbers above the columns represent the number of samples. All values are presented as mean \pm SEM. ${ }^{*} P<0.05$, ${ }^{* *} P<0.01$, ${ }^{* *} P<0.001$, ns, $P \geq 0.05$, unpaired $t$-test. Note that samples of the GFP group are also used in Fig. 1.

(Tables S3 and S4). Notably, the number of SNVs and indels of LbCas12a and AsCas12a groups were comparable to GFP ones and no significant difference was observed (Fig. 2B and 2C). For all the identified SNVs, the base substitution types showed no obvious bias (Fig. S5). Besides, the SNVs and indels observed in each embryo showed no overlap with those from other embryos (Fig. 2D). In contrast to the top predicted off-target sites, no similarity was observed between the adjacent sequences of detected off-target and the on-target sequences except for one offtarget site (Figs. S3 and S6). Notably, this off-target sequence was previously reported as a crRNA-mediated offtarget of Tp53 (Kim et al. 2016), demonstrating the sensitivity of GOAT method. We next analyzed the distribution of these SNVs in the genome context and found that they were randomly located in each chromosome, suggesting no preference for specific regions (Figs. 2E and S7). We further explored whether the SNVs and indels were enriched in transcription activated regions of the genome, where doublestrand DNA (dsDNA) are frequently unwinded to ssDNA (Zhang et al. 2012), and found that no significant difference was observed between GFP and LbCas12a or AsCas12a groups (Fig. 2F and Table S7). These results indicated that the characteristics of mutations generated in LbCas12a and AsCas12a groups were consistent with those in GFP group. Since previous studies showed that Cas12a had the targeted-activated ssDNA cleavage activity in vitro (Chen et al. 2018; Li et al. 2018). We next applied the fluorophore quencher (FQ)-labeled reporter assays to investigate the correlation between the target DNA dosage and the percentage of cleaved ssDNA (Chen et al. 2018). Our results showed that when the target DNA was diluted to $10^{-2} \mathrm{nmol} /$ $\mathrm{L}$, the ratio of cleaved ssDNA was decreased to the control level (Fig. 2G). These experiments may explain that the low amount of targeted DNA in mammalian cells leads to a relatively small ratio of target-activated ssDNA cleavage activity of Cas12a, resulting in no detectable ssDNA cleavage induced off-target effects in mouse embryos.

In summary, we developed GOAT to detect genome-wide off-target effects of gene editing tools without the need of FACS. Compared with GOTI, GOAT was a simpler and lower cost method with comparable accuracy and sensitivity. Using GOAT analysis, we found that the trans ssDNA cleavage activity of Cas12a (LbCas12a and AsCas12a) was low in mouse embryos, suggesting that Cas $12 a$ is highly specific in mammalian cells. Our results further demonstrated that the target-activated, nonspecific ssDNA cleavage activity of Cas12a in vitro was induced by a large amount of targeted dsDNA. Recent studies reported that Cas13a-mediated targeting on massive copies of RNA generated substantial "collateral effect" in cultured cells and individual organisms (Wang et al. 2019; Buchman et al. 2020). By contrast, Cas12a only targeted a limited number of gene copies in mammalian cells, and thus would not cause broad ssDNA cleavage. Besides, protective DNA repairing mechanism can repair the limited number of ssDNA cleavage (Sancar et al. 2004). In addition, low-frequency trans cleavage off-target events and large scale deletions or insertions could be missed by our detection approach, resulting in the undetectable off-target effects in our study. Considering that LbCas12a and AsCas12a have comparable editing efficiencies, smaller size and lower mismatch tolerance comparing with spCas9, they hold great promise and competition for therapeutic application in the future (Kleinstiver et al. 2016).

\section{FOOTNOTES}

This study was supported by the R\&D Program of China (2018YFC2000100 and 2017YFC1001300), the CAS Strategic Priority Research Program (XDB32060000), the National Natural Science Foundation of China (31871502, 31925016, 91957122, 31901047), the Basic Frontier Scientific Research Program of Chinese Academy of Sciences From 0 to 1 Original Innovation Project (ZDBS-LY-SM001), the Shanghai Municipal Science and Technology Major Project (2018SHZDZX05), the Shanghai City Committee of science and Technology Project (18411953700, 18JC1410100, 19XD1424400, 19YF1455100), the International Partnership Program of Chinese Academy of Sciences (153D31KYSB20170059).

The authors declare no competing interests.

The use and care of animals complied with the guideline of the Biomedical Research Ethics Committee of Shanghai Institutes for Biological Science, Chinese Academy of Sciences.

All authors reviewed and approved the final version of manuscript. 
All the sequencing data were deposited in the NCBI Sequence Read Archive (SRA) under project accession no. PRJNA637705.

Y.W. and Y.-S.Z. designed and performed experiments. Y.-J.L. R.M.L. and Q.-M.Z. performed in vitro transcription and genotyping W.-Q.Y. performed mouse embryo transfer. H.-B.Z. helped for editing the paper. C.-Y.Z., H.Y. Y.-D.S. and E.-W.Z. supervised the project and designed experiments. All the authors wrote the paper.

Yu Wei ${ }^{1,2}$, Yingsi Zhou ${ }^{1}$, Yajing Liu ${ }^{1}$, Wenqin Ying ${ }^{1}$, Ruiming Lv ${ }^{1}$, Qimeng Zhao ${ }^{1,2}$, Haibo Zhou ${ }^{1,3}$, Erwei Zuo ${ }^{4 凶}$, Yidi Sun $^{1 \bowtie}$, Hui Yang ${ }^{1,3 凶}$, Changyang Zhou ${ }^{1 凶}$

1 Institute of Neuroscience, State Key Laboratory of Neuroscience, Key Laboratory of Primate Neurobiology, Center for Excellence in Brain Science and Intelligence Technology, Chinese Academy of Sciences, Shanghai 200031, China

2 University of Chinese Academy of Sciences, Beijing 100049, China

${ }^{3}$ Shanghai Center for Brain Science and Brain-Inspired Intelligence Technology, Shanghai 201210, China

${ }^{4}$ Center for Animal Genomics, Agricultural Genome Institute at Shenzhen, Chinese Academy of Agricultural Sciences, Shenzhen 518124, China

$\triangle$ Correspondence: erweizuo@163.com (E. Zuo), sydaileen@gmail.com (Y. Sun), huiyang@ion.ac.cn (H. Yang), zhouchangyang@ion.ac.cn (C. Zhou)

Accepted January 12, 2021

\section{OPEN ACCESS}

This article is licensed under a Creative Commons Attribution 4.0 International License, which permits use, sharing, adaptation, distribution and reproduction in any medium or format, as long as you give appropriate credit to the original author(s) and the source, provide a link to the Creative Commons licence, and indicate if changes were made. The images or other third party material in this article are included in the article's Creative Commons licence, unless indicated otherwise in a credit line to the material. If material is not included in the article's Creative Commons licence and your intended use is not permitted by statutory regulation or exceeds the permitted use, you will need to obtain permission directly from the copyright holder. To view a copy of this licence, visit http:// creativecommons.org/licenses/by/4.0/.

Yu Wei, Yingsi Zhou, and Yajing Liu have contributed equally to this work.

Supplementary Information The online version of this article (https://doi.org/10.1007/s13238-021-00824-z) contains supplementary material, which is available to authorized users.

\section{REFERENCES}

Buchman A, Brogan DJ, Sun R, Yang T, Hsu P, Akbari OS (2020) Programmable RNA targeting using CasRx in flies. CRISPR J. https://doi.org/10.1089/crispr.2020.0018

Campa CC, Weisbach NR, Santinha AJ, Incarnato D, Platt RJ (2019) Multiplexed genome engineering by Cas12a and CRISPR arrays encoded on single transcripts. Nat Methods 16:887-893

Chen JS, Ma E, Harrington LB, Da Costa M, Tian X, Palefsky JM, Doudna JA (2018) CRISPR-Cas12a target binding unleashes indiscriminate single-stranded DNase activity. Science 360:436439

Gootenberg JS, Abudayyeh OO, Kellner MJ, Joung J, Collins JJ, Zhang F (2018) Multiplexed and portable nucleic acid detection platform with Cas13, Cas12a, and Csm6. Science 360:439

Gootenberg JS, Abudayyeh OO, Lee JW, Essletzbichler P, Dy AJ, Joung J, Verdine $\mathrm{V}$, Donghia $\mathrm{N}$, Daringer NM, Freije CA, Myhrvold C, Bhattacharyya RP, Livny J, Regev A, Koonin EV, Hung DT, Sabeti PC, Collins JJ, Zhang F (2017) Nucleic acid detection with CRISPR-Cas13a/C2c2. Science 356:438

Kim Y, Cheong SA, Lee JG, Lee SW, Lee MS, Baek IJ, Sung YH (2016) Generation of knockout mice by Cpf1-mediated gene targeting. Nat Biotechnol 34:808-810

Kleinstiver BP, Tsai SQ, Prew MS, Nguyen NT, Welch MM, Lopez JM, McCaw ZR, Aryee MJ, Keith Joung J (2016) Genome-wide specificities of CRISPR-Cas Cpf1 nucleases in human cells. Nat Biotechnol 34:869-874

Koo T, Park SW, Jo DH, Kim D, Kim JH, Cho H-Y, Kim J, Kim JH, Kim J-S (2018) CRISPR-LbCpf1 prevents choroidal neovascularization in a mouse model of age-related macular degeneration. Nat Commun 9:1855

Sancar A, Lindsey-Boltz LA, Ünsal-Kaçmaz K, Linn S (2004) Molecular mechanisms of mammalian DNA repair and the DNA damage checkpoints. Annu Rev Biochem 73:39-85

Li S, Cheng Q-X, Liu J-K, Nie X-Q, Zhao G-P, Wang J (2018) CRISPR-Cas12a has both cis- and trans-cleavage activities on single-stranded DNA. Cell Res 28:1

Wang Q, Liu X, Zhou J, Yang C, Wang G, Tan Y, Wu Y, Zhang S, Yi K, Kang C (2019) The CRISPR-Cas13a gene-editing system induces collateral cleavage of RNA in glioma cells. Adv Sci 6:1901299

Zetsche B, Gootenberg JS, Abudayyeh OO, Slaymaker IM, Makarova KS, Essletzbichler P, Volz SE, Joung J, van der Oost J, Regev A, Koonin EV, Zhang F (2015) Cpf1 is a single RNAguided endonuclease of a class 2 CRISPR-Cas system. Cell 163:759-771

Zhang Y, Feng Y, Chatterjee S, Tuske S, Ho MX, Arnold E, Ebright $\mathrm{RH}$ (2012) Structural basis of transcription initiation. Science (New York, NY) 338:1076-1080

Zuo E, Sun Y, Wei W, Yuan T, Ying W, Sun H, Yuan L, Steinmetz LM, Li Y, Yang H (2019) Cytosine base editor generates substantial off-target single-nucleotide variants in mouse embryos. Science 364:289-292 